Scrutiny of Prostate Cancer Antigen 3 Effectiveness as an Assist of Prostatic Specific Antigen in Screening and Early Diagnosis of Prostate

\title{
Cancer: A Review
}

\author{
Farid Azizi Jalilian,, Mohammad Soleimani, ${ }^{2, *}$ Mohsen Alemi, ${ }^{2,3}$ Arefeh Bashiri,, and Ghazaleh \\ Haghighat ${ }^{1}$ \\ ${ }^{1}$ Department of Virology, Faculty of Medicine, Hamadan University of Medical Sciences, Hamadan, Iran \\ ${ }^{2}$ Department of Urology, Faculty of Medicine, Shahid Beheshti University of Medical Sciences, Tehran, Iran \\ ${ }^{3}$ Department of Urology, Faculty of Medicine, Hamadan University of Medical Sciences, Hamadan, Iran \\ "Corresponding author: Mohammad Soleimani, Department of Urology, Shahid Modarres Hospital, Shahid Beheshti University of Medical Sciences, Tehran, Iran. Tel: \\ +98-2122074088, E-mail: mohamad.soleimani.md@gmail.com
}

Received 2017 February 21; Accepted 2017 April 19.

\begin{abstract}
PSA is the most routine marker to detect prostate cancer, but due to its low specificity that can lead to a number of unnecessary biopsies, there is great need for an alternate method. Can PCA3 be one of these methods? PCA3 is overexpressed in prostate cancer, not in benign conditions such as prostatitis (unlike PSA). Because of its efficacy (being more sensitive and specific in comparison with PSA), this biomarker could be a very useful and promising method for the early detection of prostate cancer, especially in combination with other tests such as TMPRSS2-ERG gene fusion.
\end{abstract}

Keywords: Prostatic Specific Antigen, Prostate Cancer Antigen 3, Prostate Cancer, Prostate Cancer, Screening

\section{Introduction}

Prostate cancer is the second most common malignancy in males, with an age-standardized incidence rate (ASR) of 31.1 worldwide, and the most common male cancer in Europe and the United States (1); and low in Asian populations with estimated rates of 10.5 and 4.5 in Eastern and South-Central Asia. In 2012, prostate cancer was the 5th cause of death from cancer in males (6.6\%) (2).

In a research in 5 provinces of Iran (Ardabil, Guilan, Golestan, Mazandaran, and Kerman) ASR of prostate cancer was 5.1 per 100000 person-years (3).

Now, ASR of prostate cancer is around 24.9 and 12.6, respectively in West Asia and Iran (4).

Survival of prostate cancer depends on many factors such as tumor grade, stage, invasion, and metastasis at the time of diagnosis and of course, early detection that may provide a better survival for patients (5).

Traditional approaches to screen prostate cancer (PCa) are periodic monitoring of prostate specific antigen (PSA) and digital rectal examination (DRE). PSA is a glycoprotein produced by prostate epithelial cells and for detection of prostate cancer is nonspecific (6). Thus, several studies try to find methods that increase the specificity of PSA. The most routines are percent free PSA, prostate health index (PHI), 4Kscore, and prostate cancer antigen 3 gene (PCA3) (7).

Although PSA increases in prostatic neoplasms, it can be influenced by some benign conditions such as prostatitis and benign prostatic hyperplasia (BPH). Ejaculation, prostate biopsy, and acute urinary retention can also increase PSA levels. Finasteride and dutasteride can decrease the level (8).

At a sensitivity of $80 \%$, the specificity of PSA to diagnose the prostate cancer in a cohort study, ranged between $20 \%$ and 37\% (corresponding to serum PSA values of 1.7 and 3.0 $\mathrm{ng} / \mathrm{mL}$, respectively). This low specificity (in PSA level of 3 to $15 \mathrm{ng} / \mathrm{mL}$ ), resulted in a negative biopsy rate of $70 \%$ to $80 \%$ in of the study by Hessels et al. (9). This flaw results in lots of unnecessary biopsies; therefore, there is a great need for a more specific and sensitive biomarker that can substitute PSA. According to low specificity of PSA, risk of overdiagnosis and overtreatment of PCa is notable. To face this weakness of PSA, PCa biomarkers and detection tools are used. One promising approach is to measure PCA3 in collecting urine specimens (10). 
Prostate health index (PHI) and PCA3 showed a significant increase in sensitivity and specificity, in comparison with other markers (8). In practice, there is no significant difference between the ability of PCA3 and PHI to predict PCa diagnosis in males undergoing the 1st prostate biopsy, however, combination of PCA3 and PHI is a valuable diagnosis tool (9). The higher specificity of this method can prevent unnecessary biopsies (10).

\section{Prostate Cancer Antigen 3 Gene}

PCA3 is highly overexpressed in almost all tissue specimens of prostate cancer (not in normal or hypertrophied tissue). It was 1st identified in 1999 due to collaborative research efforts by Johns Hopkins Hospital, Baltimore, and the Radboud University, Nijmegen, Netherland (11), and PCA3 was called differential display clone 3 (DD3). PCA3 is a segment of mRNA from chromosome 9q21-22'segment (12).

Multiple studies verified that PCA3 is a RNA that is not expressed outside the prostate and PCA3 levels are usually higher in cancerous tissue than benign tissue (13).

Early studies showed improved performance characteristics for PCA3 in urine over PSA to diagnose prostate cancer (14).

\section{Tanique of Sampling}

PCA3 urine samples were collected after an attentive digital rectal examination (DRE) of the prostate to exfoliate cells in urine. The initial 20 to $30 \mathrm{~mL}$ of voided urine after DRE should be collected and tested within 1 hour (15).

The PCA3 score is reported as the ratio of urine PCA3 mRNA to urine PSA mRNA $\times 1000$, normalizing PCA3 expression to PSA expression.

\section{Results of Studies}

Several experimental studies are done to evaluate the value of urine PCA3 to support as a prostate cancer biomarker, and all have presented that PCA3 scores are closely associated with the possibility of a positive biopsy (9). Moradi et al., showed that expression of this marker in urine was more sensitive than blood to distinguish cases with PCa and healthy males (16).

\section{PCA3 Score Cut-Off}

Numerous cut off points are suggested, most frequently 10, 25, and $35(17,18)$. Haese et al., described a cut off point of 35, with positive biopsy results in $39 \%$ beyond the threshold versus $22 \%$ under the threshold (17). In the
U.S. agency for healthcare quality and research, Bradley et al., indicated a threshold of 25 in a sensitivity of $74 \%$ and specificity of 57\% (19). The cut off of points of 25 was used in the food and drug administration (FDA) approval studies and 35 in the national cancer comprehensive network $(\mathrm{NCCN})$ guidelines (7).

\section{Advantages of PCA3}

A research in China showed increased PCA3 in biopsy tissue relate to prostate cancer and that the PCA3 test may enhance the diagnosis efficacy as the PCA3 level is independent of PSA score. The possibility of a positive biopsy improved with increasing PCA3 scores. The PCA3 score was significantly higher in males with prostate malignancy vs. benign prostatic hyperplasia (BPH).

The PCA3 score When PCA3, serum PSA, and biopsy Gleason score (GS) combined with each other, the diagnostic accuracy boosted to $90 \%$ to predict extracapsular extension or clinically low volume $(<0.5 \mathrm{~mL})$ tumor. Combination of PCA3 and PSA blood test can be useful to reduce overdiagnosis and adverse screening outcomes. It was proved by a cohort study by the American association for cancer research (20).

In a cohort study, 407 Italian males with a history of 2 or more PCa risk factors and at least a previous negative biopsy in urology unite of Regina Elena Cancer Institute were tested for PCA3, total PSA(tPSA), and free PSA(fPSA and f/tPSA). Out of 407 males, $48 \%$ were positive for PCa, and the PCA3 score was significantly higher among the subjects ( $P$ value $<0.0001)$. PCA3 test simplified the selection of high risk patients and had the best diagnostic interpretation, compared with tPSA and f/t PSA. Greater tumor aggressiveness correlated with higher PCA3 value (21). But another research showed that both urine PCA3 and tPSA were associated with the diagnosis of PCa. These 2 markers were independent and their combination model did not boost prediction of PCa (13). Analysis of the results of a study on the relationship between PCA3 and characteristics of tumor aggressiveness showed that PCA3 had no significant correlation with the tumor volume and primary or secondary Gleason patterns' specific tumor volume (GPTV); and PCA3 scores did not have any influence on prediction of aggressive prostate cancers (22).

In a Polish cohort prospective study, 80 males (age range 50 to 81 years) with a history of increased serum PSA level (4 to $10 \mathrm{ng} / \mathrm{mL}$ ) and no previous biopsy were tested for PCA3 (urine sample after DRE and PCA3 RNA concentration), and had prostatic biopsy on the same day. The review led to conclude that combining the result of PCA3 with PCa risk factors significantly enhanced the chance of predict- 
ing a positive prostate, compared to the results of PCA3 or PSA-alone (23).

PCA3 score correlated with grade of tumor. No link was found in association of PCA3 score alteration with age $>65$ years $(\mathrm{P}$ value $=0.975)$, family history of prostate cancer $(\mathrm{P}$ value $=0.796)$, positive DRE $(\mathrm{P}$ value $=0.179)$, use of 5-alpha-reductase inhibitors ( $P$ value $=0.793$ ), and $\mathrm{BPH} /$ prostatitis/HG-PIN/ASAP diagnosis $(\mathrm{P}$ value $=0.428)$ $(24,25)$. Compared to PSA, PCA3 was independent of prostate volume (17).

TMPRSS2-ERG gene fusion is another potential PCa biomarker, a suitable promising biomarker for its specificity to PCa tissue. The combination of TMPRSS2-ERG and PCA3 may improve diagnosis and can be used as a powerful screening method, as most of the false-negative adverse outcomes of the PCA3 test were corrected by TMPRSS2:ERG (57\%) (26).

Nearly $70 \%$ of patients with PSA value of 4 to $10 \mathrm{ng} / \mathrm{mL}$ are persuaded to undergo unnecessary prostate biopsies. Due to resistance to fluoroquinolone prophylaxis, postbiopsy infections are increasing; naturally, this problem has economical and clinical outcomes, therefore, alternative approaches should be considered. PCA3 reduces the number of unnecessary biopsies and post-biopsy infections (27). PCA3 score can remarkably prevent the number of unnecessary biopsies (32.4\% of the cases referred to Urology Unit, Cannizzaro hospital, Catania, Italy) (28).

Urine PCA3 can be used as one of the non-invasive method to diagnose prostate cancer, since it had acceptable sensitivity and specificity in a meta-analysis (29).

\section{Guidelines}

Prostate cancer early detection guideline of NCCN suggested that PCA3 seems to be most valuable to determine which patients should undertake a repeat biopsy (7). Prostate cancer guideline of European association of urology (EAU) mentioned that urine test for PCA3 is superior to total and percent free PSA to find PCa in males with elevated PSA, as it displays significant rises in the area under the receiver-operator distinctive curve for positive biopsies. PCA3 score surges with PCa size, but there are different data about whether it individually predicts GS, and its use to monitor active surveillance (AS) is unverified and confirms the comment of NCCN about the need of repeat biopsy (30).

\section{Conclusion}

Although PSA still retains its place in screening and early diagnosis of prostate cancer strongly, PCA3 test may be useful in the following PSA conditions: 1) Increased PSA with 1 or more negative biopsies to decide for repeat biopsy, 2) Large prostates, 3) Positive biopsy with normal PSA, 4) Prostatitis with increased PSA, 5) Borderline PSA in use of 5alpha-reductase inhibitors, and 6) Prediction of aggressive prostate cancers.

Since the specificity of PSA is not satisfactory, PCA3 can be used for screening and early diagnosis of prostate cancer. Combination of PCA3 with other potent biomarkers (such as TMPRSS2-ERG gene fusion, or PHI or 4Kscore) is highly recommended due to their higher ability to cover weaknesses of each other.

\section{References}

1. Torre LA, Siegel RL, Ward EM, Jemal A. Global Cancer Incidence and Mortality Rates and Trends-An Update. Cancer Epidemiol Biomarkers Prev. 2016;25(1):16-27. doi: 10.1158/1055-9965.EPI-15-0578. [PubMed: 26667886].

2. Ferlay J, Soerjomataram I, Dikshit R, Eser S, Mathers C, Rebelo M, et al. Cancer incidence and mortality worldwide: sources, methods and major patterns in GLOBOCAN 2012. Int J Cancer. 2015;136(5):E359-86. doi: 10.1002/ijc.29210. [PubMed: 25220842].

3. Sadjadi A, Nooraie M, Ghorbani A, Alimohammadian M, Zahedi MJ, Darvish-Moghadam $S$, et al. The incidence of prostate cancer in Iran: results of a population-based cancer registry. Arch Iran Med. 2007;10(4):481-5. [PubMed:17903053].

4. Roshandel G, Boreiri M, Sadjadi A, Malekzadeh R. A diversity of cancer incidence and mortality in West Asian populations. Ann Glob Health. 2014;80(5):346-57. doi: 10.1016/j.aogh.2014.09.012. [PubMed: 25512150].

5. Rahal AK, Badgett RG, Hoffman RM. Screening Coverage Needed to Reduce Mortality from Prostate Cancer: A Living Systematic Review.PLoS One. 2016;11(4):e0153417. doi: 10.1371/journal.pone.0153417. [PubMed: 27070904].

6. Daskivich TJ, Fan KH, Koyama T, Albertsen PC, Goodman M, Hamilton AS, et al. Prediction of long-term other-cause mortality in men with early-stage prostate cancer: results from the Prostate Cancer Outcomes Study. Urology. 2015;85(1):92-100. doi: 10.1016/j.urology.2014.07.003. [PubMed: 25261048].

7. Prostate Cancer Early Detection. . National cancer comprehensive network clinical practice guidelines in oncology 2016. [cited 9 September]. Available from: https://www.nccn.org/professionals/ physician_gls/pdf/prostate_detection.pdf.

8. Mottet N, Bellmunt J, Briers E. Eau-estro-siog guidelines on prostate cancer. Europ Association Urol. .

9. Hessels D, Verhaegh GW, Schalken JA, Witjes JA. Applicability of biomarkers in the early diagnosis of prostate cancer. Expert Rev Mol Diagn. 2004;4(4):513-26. doi: 10.1586/14737159.4.4.513. [PubMed: 15225099].

10. Truong M, Yang B, Jarrard DF. Toward the detection of prostate cancer in urine: a critical analysis. J Urol. 2013;189(2):422-9. doi: 10.1016/j.juro.2012.04.143. [PubMed: 23017522].

11. Bussemakers MJ, van Bokhoven A, Verhaegh GW, Smit FP, Karthaus HF, Schalken JA, et al. DD3: a new prostate-specific gene, highly overexpressed in prostate cancer. Cancer Res. 1999;59(23):5975-9. [PubMed: 10606244].

12. Bourdoumis A, Papatsoris AG, Chrisofos M, Efstathiou E, Skolarikos A, Deliveliotis C. The novel prostate cancer antigen 3 (PCA3) biomarker. Int Braz J Urol. 2010;36(6):665-8. [PubMed: 21176272] discussion 669. 
13. de Kok JB, Verhaegh GW, Roelofs RW, Hessels D, Kiemeney LA, Aalders TW, et al. DD3(PCA3), a very sensitive and specific marker to detect prostate tumors. Cancer Res. 2002;62(9):2695-8. [PubMed: 11980670].

14. Hessels D, Smit FP, Verhaegh GW, Witjes JA, Cornel EB, Schalken JA. Detection of TMPRSS2-ERG fusion transcripts and prostate cancer antigen 3 in urinary sediments may improve diagnosis of prostate cancer. Clin Cancer Res. 2007;13(17):5103-8. doi: 10.1158/1078-0432.CCR-070700. [PubMed: 17785564].

15. Sokoll LJ, Ellis W, Lange P, Noteboom J, Elliott DJ, Deras IL, et al. A multicenter evaluation of the PCA3 molecular urine test: pre-analytical effects, analytical performance, and diagnostic accuracy. Clin Chim Acta. 2008;389(1-2):1-6. doi: 10.1016/j.cca.2007.11.003. [PubMed: 18061575].

16. Moradi Sardareh H, Goodarzi MT, Yadegar-Azari R, Poorolajal J, Mousavi-Bahar SH, Saidijam M. Prostate cancer antigen 3 gene expression in peripheral blood and urine sediments from prostate cancer and benign prostatic hyperplasia patients versus healthy individuals. Urol J. 2014;11(6):1952-8. [PubMed: 25433473].

17. Haese A, de la Taille A, van Poppel H, Marberger M, Stenzl A, Mulders PF, et al. Clinical utility of the PCA3 urine assay in European men scheduled for repeat biopsy. Eur Urol. 2008;54(5):1081-8. doi: 10.1016/j.eururo.2008.06.071. [PubMed:18602209].

18. Crawford ED, Rove KO, Trabulsi EJ, Qian J, Drewnowska KP, Kaminetsky JC, et al. Diagnostic performance of PCA3 to detect prostate cancer in men with increased prostate specific antigen: a prospective study of 1,962 cases. J Urol. 2012;188(5):1726-31. doi: 10.1016/j.juro.2012.07.023. [PubMed: 22998901].

19. Bradley LA, Palomaki GE, Gutman S, Samson D, Aronson N. Comparative effectiveness review: prostate cancer antigen 3 testing for the diagnosis and management of prostate cancer. JUrol. 2013;190(2):38998. doi: 10.1016/j.juro.2013.02.005. [PubMed: 23545099].

20. Birnbaum JK, Feng Z, Gulati R, Fan J, Lotan Y, Wei JT, et al. Projecting Benefits and Harms of Novel Cancer Screening Biomarkers: A Study of PCA3 and Prostate Cancer. Cancer Epidemiol Biomarkers Prev. 2015;24(4):677-82. doi: 10.1158/1055-9965.EPI-14-1224. [PubMed: 25613117].
21. Merola R, Tomao L, Antenucci A, Sperduti I, Sentinelli S, Masi S, et al. PCA3 in prostate cancer and tumor aggressiveness detection on 407 high-risk patients: a National Cancer Institute experience. J Experimental Clinical Cancer Res. 2015;34(1):15. doi:10.1186/s13046-015-0127-8.

22. Augustin H, Mayrhofer K, Pummer K, Mannweiler S. Relationship between prostate cancer gene 3 (PCA3) and characteristics of tumor aggressiveness. Prostate. 2013;73(2):203-10. doi: 10.1002/pros.22558. [PubMed: 22782916].

23. Salaglerski M, Mulders P, Schalken JA. Predicting prostate biopsy outcome using a PCA3-based nomogram in a polish cohort. Anticancer Res. 2013;33:553-8.

24. De Luca S, Passera R, Cappia S, Bollito E, Randone DF, Porpiglia F. Pathological patterns of prostate biopsy in men with fluctuations of prostate cancer gene 3 score: a preliminary report. Anticancer Res. 2015;35(4):2417-22. [PubMed: 25862908].

25. De Luca S, Passera R, Cappia S, Bollito E, Randone DF, Milillo A, et al Fluctuation in prostate cancer gene 3(PCA3) score in men undergoing first or repeat prostate biopsies. BJU Int. 2014.

26. Robert G, Jannink S, Smit F, Aalders T, Hessels D, Cremers R, et al Rational basis for the combination of PCA3 and TMPRSS2:ERG gene fusion for prostate cancer diagnosis. Prostate. 2013;73(2):113-20. doi: 10.1002/pros.22546. [PubMed: 22674214].

27. Batura D, Gopal Rao G. The national burden of infections af ter prostate biopsy in England and Wales: a wake-up call for better prevention. J Antimicrob Chemother. 2013;68(2):247-9. doi: 10.1093/jac/dks401. [PubMed: 23047808].

28. Pepe P, Aragona F. PCA3 score vs PSA free/total accuracy in prostate cancer diagnosis at repeat saturation biopsy. Anticancer Res. 2011;31(12):4445-9. [PubMed: 22199313].

29. Xue WJ, Ying XL, Jiang JH, Xu YH. Prostate cancer antigen 3 as a biomarker in the urine for prostate cancer diagnosis: a meta-analysis. J Cancer Res Ther. 2014;10 Suppl:C218-21. doi: 10.4103/0973-1482.145881. [PubMed: 25450286].

30. Mottet N, Bellmunt J, Briers E. Eau-estro-siog guidelines on prostate cancer. Europ Association Urol. 2016. 